\title{
The existence of optimal approximate solution theorems for generalized $\alpha$-proximal contraction non-self mappings and applications
}

\author{
Erdal Karapınar ${ }^{1 *}$ and Wutiphol Sintunavarat ${ }^{2 *}$
}

\author{
"Correspondence: \\ erdalkarapinar@yahoo.com; \\ ekarapinar@atilim.edu.tr; \\ wutiphol@mathstat.sci.tu.ac.th; \\ poom_teun@hotmail.com \\ ${ }^{1}$ Department of Mathematics, \\ Faculty of Science and Art, Atilim \\ University, İncek, Ankara 06836, \\ Turkey \\ 2 Department of Mathematics and \\ Statistics, Faculty of Science and \\ Technology, Thammasat University \\ Rangsit Center, Pathumthani, 12121, \\ Thailand
}

\begin{abstract}
In this paper, we investigate the sufficient conditions to find a best proximity point for a certain class of non-self mappings. It is well known that optimization problems can be transformed to the problems of the existence of a best proximity point. Hence, improvement in the best proximity point theory implicitly develops the theory of optimization. Our presented results generalize, extent and improve various well-known results on the topic in the literature. In particular, we consider some applications of our results to the best proximity point theorems on a class of metric spaces endowed with an arbitrary binary relation which involves the partially ordered metric spaces.
\end{abstract}

MSC: $47 \mathrm{H} 10 ; 54 \mathrm{H} 25$

Keywords: approximately compact; best proximity point; binary relation; generalized $\alpha$-proximal contraction of the first kind; generalized $\alpha$-proximal contraction of the second kind

\section{Introduction and preliminaries}

Various nonlinear problems arising in several branches of mathematics, besides some other quantitative sciences such as economics, biology, physics and engineering, can be transformed to a fixed point problem of the form $T x=x$ for a self-mapping $T$ defined on a subset of the metric space $(X, d)$. Among them, optimization problems, differential equation problems, integral equation problems, variational problems, equilibrium problems have attracted attention of researchers. The renowned Banach contraction principle of Banach [1] is a very crucial and popular tool for solving fixed point problems in the setting of a self-mapping $T$. On the other hand, if $T$ is not a self-mapping ( $T: A \rightarrow B$ where $A$ and $B$ are non-empty subsets of a metric space $X$ ), then $T$ does not necessarily have a fixed point. Therefore, the equation $T x=x$ could have no solution. In this case, it is natural to search for a point $x \in A$ in a way that the distance between $x$ and its image $T x$ is as small as possible. In this discussion, the best approximation theorem plays an important role in studying the existence of an approximate solution that is optimal for the equation $T x=x$.

Let $A, B$ be subsets of the metric space $(X, d)$. Under the setting of $d(x, T x)=d(A, B):=$ $\inf \{d(x, y): x \in A$ and $y \in B\}, x$ is called a best proximity point. Notice that the point $x \in A$ is the global minimum of the error involved for an approximate solution of the equation

(O2013 Karapınar and Sintunavarat; licensee Springer. This is an Open Access article distributed under the terms of the Creative Commons Attribution License (http://creativecommons.org/licenses/by/2.0), which permits unrestricted use, distribution, and reproduction in any medium, provided the original work is properly cited. 
$T x=x$. Therefore, we conclude that the purpose of the existence theorems for a best proximity point is to provide sufficient conditions to solve a minimization problem. One of the initial and most interesting best approximation theorems in this direction was given by Fan [2]. Following this result, several authors have reported a number of results in this field; see, e.g., [3-14] and the references therein.

The notion of approximate compactness, introduced by Efimov and Stechkin [15], plays an important role in the theory of approximation. The properties of approximately compact sets have been studied intensively. The following definition is a generalization of this concept.

Definition 1.1 Let $A$ and $B$ be two non-empty subsets of the metric space $(X, d)$. Then $B$ is said to be approximately compact with respect to $A$ if every sequence $\left\{y_{n}\right\}$ of $B$, satisfying the condition $d\left(x, y_{n}\right) \rightarrow d(x, B)$ as $n \rightarrow \infty$ for some $x \in A$, has a convergent subsequence.

Remark 1.1 Any non-empty subset of the metric space $(X, d)$ is approximately compact with respect to itself. Moreover, if $B$ is compact, then $B$ is approximately compact with respect to $A$.

On the other hand, Samet et al. [16] introduced the concept of $\alpha$-admissible mapping as follows.

Definition 1.2 [16] Let $X$ be a non-empty set, $T: X \rightarrow X$ and $\alpha: X \times X \rightarrow[0, \infty)$. We say that $T$ is an $\alpha$-admissible if for all $x, y \in X$, we have

$$
\alpha(x, y) \geq 1 \quad \Longrightarrow \quad \alpha(T x, T y) \geq 1
$$

Using this concept, they proved some fixed point theorems. The authors also showed that these results can be utilized to derive fixed point theorems in partially ordered spaces. Moreover, they applied their main results to solve certain types of ordinary differential equations. Afterward, a number of papers have reported on fixed point theory with applications to ordinary differential equations via the concept of $\alpha$-admissible mapping in various directions (see [17-22] and references therein).

Recently, Jleli and Samet [23] introduced the notion of $\alpha$-proximal admissible mapping which is a non-self version of an $\alpha$-admissible mapping.

Definition 1.3 [23] Let $A$ and $B$ be two non-empty subsets of the metric space $(X, d)$ and $\alpha: A \times A \rightarrow[0, \infty)$. A mapping $T: A \rightarrow B$ is said to be $\alpha$-proximal admissible if for all $u, v, x, y \in A$,

$$
\left\{\begin{array}{l}
\alpha(x, y) \geq 1, \\
d(u, T x)=d(A, B), \quad \Longrightarrow \quad \alpha(u, v) \geq 1 . \\
d(v, T y)=d(A, B),
\end{array}\right.
$$

Remark 1.2 If $A=B$, then the notion of $\alpha$-proximal admissibility $T$ coincides with the concept of $\alpha$-admissibility. 
Inspired by [23], we introduce the class of non-self mappings, generalized $\alpha$-proximal contraction mappings. Also, we establish the existence theorems for a best proximity point for mappings in this class under the assumptions of approximate compactness and nonapproximate compactness of subspaces. Our presented results generalize, extent and improve various well-known results such as the results of Banach [1], Kannan [24], Chatterjea [25], Berinde [26]. As an application, we apply our results to the existence theorems for a best proximity point on a metric space endowed with an arbitrary binary relation. Furthermore, we give the special case of these results in partially ordered metric spaces.

\section{Best proximity point theorems for an $\alpha$-proximal contraction non-self mapping}

In the sequel, unless otherwise specified, $\mathbb{R}$ and $\mathbb{N}$ denote the set of real numbers and the set of positive integers, respectively.

Let $A$ and $B$ be two non-empty subsets of a metric space $(X, d)$. The following notations will be used in the sequel:

$$
\begin{aligned}
& d(A, B):=\inf \{d(x, y): x \in A \text { and } y \in B\}, \\
& A_{0}:=\{x \in A: d(x, y)=d(A, B) \text { for some } y \in B\}, \\
& B_{0}:=\{y \in B: d(x, y)=d(A, B) \text { for some } x \in A\} .
\end{aligned}
$$

For a non-self mapping $T: A \rightarrow B$, we define the set of all best proximity points of $T$ by $\mathcal{B}_{\text {est }}(T)$, that is,

$$
\mathcal{B}_{\text {est }}(T):=\{x \in A: d(x, T x)=d(A, B)\} .
$$

Remark 2.1 Kirk et al. [27] gave sufficient conditions to ensure that $A_{0}$ and $B_{0}$ are nonempty. Also, we obtain that if $A$ and $B$ are closed subsets of a normed linear space such that $d(A, B)>0$, then $A_{0}$ and $B_{0}$ are contained in the boundaries of $A$ and $B$, respectively (see [28]).

Now, we introduce new classes of generalized proximal contraction non-self mappings.

Definition 2.1 Let $A$ and $B$ be two non-empty subsets of a metric space $(X, d)$ and $\alpha$ : $A \times A \rightarrow[0, \infty)$. A mapping $T: A \rightarrow B$ is said to be a generalized $\alpha$-proximal contraction of the first kind if there exist non-negative real numbers $\theta_{1}, \theta_{2}, \theta_{3}$ and $L$ with $\theta_{1}+2 \theta_{2}+2 \theta_{3}<1$ such that for all $u, v, x, y \in A$,

$$
\left\{\begin{array}{llrl}
\alpha(x, y) \geq 1, & d(u, v) \leq & \theta_{1} d(x, y)+\theta_{2}[d(x, u)+d(y, v)] \\
d(u, T x)=d(A, B), & & +\theta_{3}[d(x, v)+d(y, u)] \\
d(v, T y)=d(A, B), & & & L \min \{d(y, u), d(x, v), d(x, u), d(y, v)\} .
\end{array}\right.
$$

Definition 2.2 Let $A$ and $B$ be two non-empty subsets of the metric space $(X, d)$ and $\alpha$ : $A \times A \rightarrow[0, \infty)$. A mapping $T: A \rightarrow B$ is said to be a generalized $\alpha$-proximal contraction of the second kind if there exist non-negative real numbers $\theta_{1}, \theta_{2}, \theta_{3}$ and $L$ with $\theta_{1}+2 \theta_{2}+2 \theta_{3}<$ 
1 such that for all $u, v, x, y \in A$,

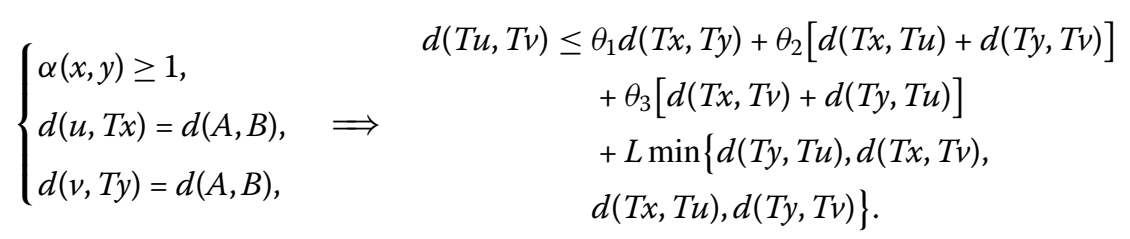

Next, we give our first main result which is the best proximity point theorem for a generalized $\alpha$-proximal contraction.

Theorem 2.1 Let $(X, d)$ be a complete metric space, and let $A$ and $B$ be two non-empty, closed subsets of $X$ such that $B$ is approximately compact with respect to $A$. Assume that $\alpha$ : $A \times A \rightarrow[0, \infty), A_{0}$ and $B_{0}$ are non-empty sets and $T: A \rightarrow B$ is a generalized $\alpha$-proximal contraction of the first kind such that the following conditions hold:

(a) $T$ is $\alpha$-proximal admissible;

(b) $T\left(A_{0}\right) \subseteq B_{0}$;

(c) There exist $x_{0}, x_{1} \in A$ such that $d\left(x_{1}, T x_{0}\right)=d(A, B)$ and $\alpha\left(x_{0}, x_{1}\right) \geq 1$;

(d) If $\left\{x_{n}\right\}$ is a sequence in $A$ such that $\alpha\left(x_{n}, x_{n+1}\right) \geq 1$ for all $n \in \mathbb{N}$ and $x_{n} \rightarrow x$ as $n \rightarrow \infty$ for some $x \in A$, then $\alpha\left(x_{n}, x\right) \geq 1$ for all $n \in \mathbb{N}$.

Then there exists an element $z \in A_{0}$ such that $d(z, T z)=d(A, B)$, that is, $T$ has at least one best proximity point. Moreover, if $\alpha\left(z_{1}, z_{2}\right) \geq 1$ for all $z_{1}, z_{2} \in \mathcal{B}_{\text {est }}(T)$, then $T$ has a unique best proximity point.

Proof From (c), there exist $x_{0}, x_{1} \in A$ such that

$$
d\left(x_{1}, T x_{0}\right)=d(A, B)
$$

and

$$
\alpha\left(x_{0}, x_{1}\right) \geq 1 .
$$

Since $T\left(A_{0}\right) \subseteq B_{0}$, then by the definition of $B_{0}$, there exists $x_{2} \in A_{0}$ such that

$$
d\left(x_{2}, T x_{1}\right)=d(A, B) .
$$

Since $T$ is $\alpha$-proximal admissible, by (2.1), (2.2) and (2.3), we have

$$
\alpha\left(x_{1}, x_{2}\right) \geq 1
$$

Again, since $T\left(A_{0}\right) \subseteq B_{0}$, there exists $x_{3} \in A_{0}$ such that

$$
d\left(x_{3}, T x_{2}\right)=d(A, B) .
$$

Using (2.3), (2.4), (2.5) and the assumption that $T$ is $\alpha$-proximal admissible, we get

$$
\alpha\left(x_{2}, x_{3}\right) \geq 1 .
$$


Continuing this process, we can construct a sequence $\left\{x_{n}\right\}$ in $A_{0}$ such that

$$
d\left(x_{n}, T x_{n-1}\right)=d(A, B)
$$

and

$$
\alpha\left(x_{n-1}, x_{n}\right) \geq 1
$$

for all $n \in \mathbb{N}$. Since $T$ is a generalized $\alpha$-proximal contraction of the first kind, for each $n \in \mathbb{N}$, we have

$$
\begin{aligned}
d\left(x_{n}, x_{n+1}\right) \leq & \theta_{1} d\left(x_{n-1}, x_{n}\right)+\theta_{2}\left[d\left(x_{n-1}, x_{n}\right)+d\left(x_{n}, x_{n+1}\right)\right]+\theta_{3}\left[d\left(x_{n-1}, x_{n+1}\right)+d\left(x_{n}, x_{n}\right)\right] \\
& +L \min \left\{d\left(x_{n}, x_{n}\right), d\left(x_{n-1}, x_{n+1}\right), d\left(x_{n-1}, x_{n}\right), d\left(x_{n}, x_{n+1}\right)\right\} \\
\leq & \theta_{1} d\left(x_{n-1}, x_{n}\right)+\theta_{2}\left[d\left(x_{n-1}, x_{n}\right)+d\left(x_{n}, x_{n+1}\right)\right]+\theta_{3}\left[d\left(x_{n-1}, x_{n}\right)+d\left(x_{n}, x_{n+1}\right)\right] .
\end{aligned}
$$

It follows that $d\left(x_{n}, x_{n+1}\right) \leq K d\left(x_{n-1}, x_{n}\right)$ for all $n \in \mathbb{N}$, where $K:=\frac{\theta_{1}+\theta_{2}+\theta_{3}}{1-\theta_{2}-\theta_{3}} \in[0,1)$. By induction we get

$$
d\left(x_{n}, x_{n+1}\right) \leq K^{n} d\left(x_{0}, x_{1}\right)
$$

for all $n \in \mathbb{N}$. It easily follows that for $m, n \in \mathbb{N}$ such that $m<n$,

$$
\begin{aligned}
d\left(x_{m}, x_{n}\right) & \leq \sum_{i=m}^{n-1} d\left(x_{i}, x_{i+1}\right) \\
& \leq\left(K^{m}+K^{m+1}+\cdots+K^{n-1}\right) d\left(x_{0}, x_{1}\right) \\
& \leq \frac{K^{m}}{1-h} d\left(x_{0}, x_{1}\right) .
\end{aligned}
$$

This implies that $\left\{x_{n}\right\}$ is a Cauchy sequence. Now, since $X$ is complete and $A$ is closed, the sequence $\left\{x_{n}\right\}$ converges to some $z \in A$. Further, we have

$$
\begin{aligned}
d(z, B) & \leq d\left(z, T x_{n}\right) \\
& \leq d\left(z, x_{n+1}\right)+d\left(x_{n+1}, T x_{n}\right) \\
& =d\left(z, x_{n+1}\right)+d(A, B) \\
& \leq d\left(z, x_{n+1}\right)+d(z, B)
\end{aligned}
$$

for all $n \in \mathbb{N}$. Therefore, $d\left(z, T x_{n}\right) \rightarrow d(z, B)$ as $n \rightarrow \infty$. Since $B$ is approximately compact with respect to $A$, then the sequence $\left\{T x_{n}\right\}$ has a subsequence $\left\{T x_{n_{k}}\right\}$ converging to some element $w \in B$. Therefore, $d(z, w)=\lim _{k \rightarrow \infty} d\left(x_{n_{k}+1}, T x_{n_{k}}\right)=d(A, B)$, and hence $z$ must be a member of $A_{0}$. Because of the fact that $T\left(A_{0}\right)$ is contained in $B_{0}, d(u, T z)=d(A, B)$ for some element $u$ in $A$.

From the hypothesis (d) and (2.8), we get

$$
\alpha\left(x_{n}, z\right) \geq 1
$$


for all $n \in \mathbb{N}$. Now, we have

$$
\left\{\begin{array}{l}
\alpha\left(x_{n}, z\right) \geq 1, \\
d\left(x_{n+1}, T x_{n}\right)=d(A, B), \\
d(u, T z)=d(A, B),
\end{array}\right.
$$

for all $n \in \mathbb{N}$. Since $T$ is a generalized $\alpha$-proximal contraction of the first kind, we get

$$
\begin{aligned}
d\left(x_{n+1}, u\right) \leq & \theta_{1} d\left(x_{n}, z\right)+\theta_{2}\left[d(u, z)+d\left(x_{n+1}, x_{n}\right)\right]+\theta_{3}\left[d\left(x_{n+1}, z\right)+d\left(u, x_{n}\right)\right] \\
& +L \min \left\{d\left(u, x_{n}\right), d\left(x_{n+1}, z\right), d(u, z), d\left(x_{n+1}, x_{n}\right)\right\}
\end{aligned}
$$

for all $n \in \mathbb{N}$. Taking the limit as $n \rightarrow \infty$ in the above inequality, we get $z=u$. Thus, it follows that $d(z, T z)=d(u, T z)=d(A, B)$. Therefore, $z$ is at least one of the best proximity points of $T$.

Finally, we prove the uniqueness of the best proximity point. Assume that $\tilde{z}$ is another best proximity point of $T$ such that $\alpha(z, \tilde{z}) \geq 1$, then we have

$$
\left\{\begin{array}{l}
\alpha(z, \tilde{z}) \geq 1, \\
d(z, T z)=d(A, B), \\
d(\tilde{z}, T \tilde{z})=d(A, B) .
\end{array}\right.
$$

Again, since $T$ is a generalized $\alpha$-proximal contraction of the first kind, we obtain that

$$
\begin{aligned}
d(z, \tilde{z}) \leq & \theta_{1} d(z, \tilde{z})+\theta_{2}[d(z, z)+d(\tilde{z}, \tilde{z})]+\theta_{3}[d(z, \tilde{z})+d(\tilde{z}, z)] \\
& +L \min \{d(\tilde{z}, z), d(z, \tilde{z}), d(z, z), d(\tilde{z}, \tilde{z})\}
\end{aligned}
$$

which implies that $d(z, \tilde{z})=0$, and then $z=\tilde{z}$. Hence $T$ has a unique best proximity point. This completes the proof.

Now we give some examples to support our result.

Example 2.2 Let $X=\mathbb{R}$ with the usual metric $d: X \times X \rightarrow[0, \infty)$ defined by $d(x, y):=$ $|x-y|$. Clearly, $(X, d)$ is a complete metric space. Also, let $A=(-\infty,-2]$ and $B=\left[\frac{1}{3}, 200\right]$ be two closed subsets of $X$. It is obtained that $B$ is compact and so $B$ is approximately compact with respect to $A$. Define a non-self mapping $T: A \rightarrow B$ by

$$
T x= \begin{cases}\frac{1}{2}, & x \in(-\infty,-5), \\ \frac{1}{3}-x^{3}, & x \in[-5,-3), \\ -\frac{1}{5} x-\frac{1}{15}, & x \in[-3,-2] .\end{cases}
$$

Clearly, $d(A, B)=\frac{7}{3}$ and

$$
\begin{aligned}
& A_{0}=\left\{x \in A: d(x, y)=d(A, B)=\frac{7}{3} \text { for some } y \in B\right\}=\{-2\} \\
& B_{0}=\left\{y \in B: d(x, y)=d(A, B)=\frac{7}{3} \text { for some } x \in A\right\}=\left\{\frac{1}{3}\right\} .
\end{aligned}
$$


It is easy to see that $T\left(A_{0}\right) \subseteq B_{0}$. Define $\alpha: A \times A \rightarrow[0, \infty)$ by

$$
\alpha(x, y)= \begin{cases}e^{|x-y|}, & x, y \in[-3,-2] \\ 0, & \text { otherwise. }\end{cases}
$$

Now we show that $T$ is a generalized $\alpha$-proximal contraction of the first kind. Assume that $u, v, x, y \in A$ such that

$$
\left\{\begin{array}{l}
\alpha(x, y) \geq 1, \\
d(u, T x)=d(A, B), \\
d(v, T y)=d(A, B) .
\end{array}\right.
$$

Then

$$
\left\{\begin{array}{l}
x, y \in[-3,-2] \\
d(u, T x)=\frac{7}{3} \\
d(v, T y)=\frac{7}{3}
\end{array}\right.
$$

Since $T z \in\left[\frac{1}{3}, \frac{8}{15}\right]$ for all $z \in[-3,-2]$, we get $u=v=-2$. Therefore,

$$
\begin{aligned}
0=d(u, v) \leq & \theta_{1} d(x, y)+\theta_{2}[d(x, u)+d(y, v)]+\theta_{3}[d(x, v)+d(y, u)] \\
& +L \min \{d(y, u), d(x, v), d(x, u), d(y, v)\}
\end{aligned}
$$

where $\theta_{1}, \theta_{2}, \theta_{3}$ and $L$ are non-negative real numbers with $\theta_{1}+2 \theta_{2}+2 \theta_{3}<1$. This shows that $T$ is a generalized $\alpha$-proximal contraction of the first kind.

Next, we prove that $T$ is $\alpha$-proximal admissible. Assume that $u, v, x, y \in A$ such that

$$
\left\{\begin{array}{l}
\alpha(x, y) \geq 1, \\
d(u, T x)=d(A, B), \\
d(v, T y)=d(A, B) .
\end{array}\right.
$$

Then we have $x, y \in[-3,-2]$ and $u=v=-2$. So $\alpha(u, v)=\alpha(-2,-2)=1$ and thus $T$ is $\alpha$-proximal admissible.

It is easy to see that there exist $x_{0}, x_{1} \in A$ such that $d\left(x_{1}, T x_{0}\right)=d(A, B)$ and $\alpha\left(x_{0}, x_{1}\right) \geq 1$. Assume that $\left\{x_{n}\right\}$ is a sequence in $A$ such that $\alpha\left(x_{n}, x_{n+1}\right) \geq 1$ for all $n \in \mathbb{N}$ and $x_{n} \rightarrow x$ as $n \rightarrow \infty$. Therefore, $x_{n} \in[-3,-2]$ for all $n \in \mathbb{N}$. By the closeness of [-3,-2], we get $x \in$ $[-3,-2]$ and hence $\alpha\left(x_{n}, x\right) \geq 1$ for all $n \in \mathbb{N}$.

Consequently, all the hypotheses of Theorem 2.1 are satisfied and so $T$ has at least one best proximity point, that is, a point -2 such that

$$
d(-2, T(-2))=d\left(-2, \frac{1}{3}\right)=\frac{7}{3}=d(A, B) .
$$

Example 2.3 Let $X=\mathbb{R}$ with the usual metric $d: X \times X \rightarrow[0, \infty)$ defined by $d(x, y):=$ $|x-y|$. Clearly, $(X, d)$ is a complete metric space. Also, let $A=[-1,1] \cup\{5,10\}$ and $B=$ 
$\left[-\frac{1}{3}, \frac{1}{3}\right] \cup\{5,10\}$ be two closed subsets of $X$. It is obtained that $B$ is compact and so $B$ is approximately compact with respect to $A$. Define a non-self mapping $T: A \rightarrow B$ by

$$
T x= \begin{cases}\frac{1}{3} x, & x \in[-1,1], \\ 0, & x=5, \\ 5, & x=10 .\end{cases}
$$

Clearly, $d(A, B)=0$ and

$$
\begin{aligned}
& A_{0}=\{x \in A: d(x, y)=d(A, B)=0 \text { for some } y \in B\}=B, \\
& B_{0}=\{y \in B: d(x, y)=d(A, B)=0 \text { for some } x \in A\}=B .
\end{aligned}
$$

It is easy to see that $T\left(A_{0}\right) \subseteq B_{0}$. Define $\alpha: A \times A \rightarrow[0, \infty)$ by

$$
\alpha(x, y)= \begin{cases}x^{2}+y^{2}+\pi, & x, y \in[-1,1] \\ 0, & \text { otherwise. }\end{cases}
$$

Now we show that $T$ is a generalized $\alpha$-proximal contraction of the first kind with $\theta_{1}=\frac{1}{2}$, $\theta_{2}=\frac{1}{10}, \theta_{3}=\frac{1}{10}$ and $L=2$. Assume that $u, v, x, y \in A$ such that

$$
\left\{\begin{array}{l}
\alpha(x, y) \geq 1, \\
d(u, T x)=d(A, B), \\
d(v, T y)=d(A, B) .
\end{array}\right.
$$

Then

$$
\left\{\begin{array}{l}
x, y \in[-1,1] \\
d(u, T x)=0 \\
d(v, T y)=0 .
\end{array}\right.
$$

Hence, $u=T x=\frac{1}{3} x$ and $v=T y=\frac{1}{3} y$. Therefore,

$$
\begin{aligned}
d(u, v)= & d(T x, T y) \\
= & \frac{1}{3}|x-y| \\
\leq & \frac{1}{2} d(x, y)+\frac{1}{10}[d(x, u)+d(y, v)]+\frac{1}{10}[d(x, v)+d(y, u)] \\
& +2 \min \{d(y, u), d(x, v), d(x, u), d(y, v)\} .
\end{aligned}
$$

This shows that $T$ is a generalized $\alpha$-proximal contraction of the first kind.

Next, we prove that $T$ is $\alpha$-proximal admissible. Assume that $u, v, x, y \in A$ such that

$$
\left\{\begin{array}{l}
\alpha(x, y) \geq 1, \\
d(u, T x)=d(A, B), \\
d(v, T y)=d(A, B) .
\end{array}\right.
$$


Then we have $x, y \in[-1,1]$ and so

$$
u=T x \in\left[-\frac{1}{3}, \frac{1}{3}\right] \subseteq[-1,1]
$$

and

$$
v=T y \in\left[-\frac{1}{3}, \frac{1}{3}\right] \subseteq[-1,1] .
$$

Thus $\alpha(u, v)=\alpha(T x, T y) \geq 1$. This implies that $T$ is $\alpha$-proximal admissible.

It is easy to see that there exist $x_{0}, x_{1} \in A$ such that $d\left(x_{1}, T x_{0}\right)=d(A, B)$ and $\alpha\left(x_{0}, x_{1}\right) \geq 1$.

Suppose that $\left\{x_{n}\right\}$ is a sequence in $A$ such that $\alpha\left(x_{n}, x_{n+1}\right) \geq 1$ for all $n \in \mathbb{N}$ and $x_{n} \rightarrow x$ as $n \rightarrow \infty$. Therefore, $x_{n} \in[-1,1]$ for all $n \in \mathbb{N}$. Since $[-1,1]$ is closed, we get $x \in[-1,1]$ and hence $\alpha\left(x_{n}, x\right) \geq 1$ for all $n \in \mathbb{N}$.

Consequently, all the hypotheses of Theorem 2.1 are satisfied and so $T$ has at least one best proximity point, that is, a point 0 such that

$$
d(0, T(0))=d(A, B)
$$

It is easy to see that Theorem 2.1 yields the following corollary.

Corollary 2.4 Let $(X, d)$ be a complete metric space, and let $A$ and $B$ be two non-empty, closed subsets of $X$ such that $B$ is approximately compact with respect to A. Assume that $A_{0}$ and $B_{0}$ are non-empty sets and $T: A \rightarrow B$ satisfies the following conditions:

$\left(\mathrm{a}^{\prime}\right)$ There exist non-negative real numbers $\theta_{1}, \theta_{2}, \theta_{3}$ and $L$ with $\theta_{1}+2 \theta_{2}+2 \theta_{3}<1$ such that for all $u, v, x, y \in A$,

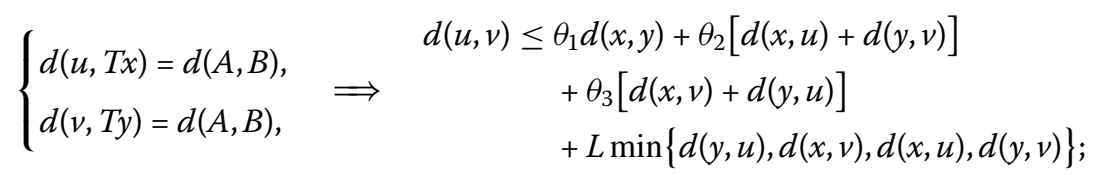

(b) $T\left(A_{0}\right) \subseteq B_{0}$;

(c) There exist $x_{0}, x_{1} \in A$ such that $d\left(x_{1}, T x_{0}\right)=d(A, B)$.

Then there exists unique $z \in A_{0}$ such that $d(z, T z)=d(A, B)$, that is, $T$ has a unique best proximity point.

Proof By taking $\alpha(x, y)=1$ for all $x, y \in A$ in Theorem 2.1, we get this result.

In Corollary 2.4, if $T$ is a self-mapping, then we get the following fixed point theorem.

Corollary 2.5 Let $(X, d)$ be a complete metric space, $A$ be a non-empty, closed subset of $X$ and $T: A \rightarrow A$. If there exist non-negative real numbers $\theta_{1}, \theta_{2}, \theta_{3}$ and $L$ with $\theta_{1}+2 \theta_{2}+2 \theta_{3}<$ 1 such that

$$
\begin{aligned}
d(T x, T y) \leq & \theta_{1} d(x, y)+\theta_{2}[d(x, T x)+d(y, T y)]+\theta_{3}[d(x, T y)+d(y, T x)] \\
& +L \min \{d(y, T x), d(x, T v), d(x, T x), d(y, T y)\}
\end{aligned}
$$

for all $x, y \in A$, then $T$ has a unique fixed point. 


\section{Remark 2.2}

1. If $\theta_{2}=\theta_{3}=L=0$ in Corollary 2.5, we get the generalized and improved result of Banach [1].

2. If $\theta_{1}=\theta_{3}=L=0$ in Corollary 2.5, we get the generalized and improved result of Kannan [24].

3. If $\theta_{1}=\theta_{2}=L=0$ in Corollary 2.5, we get the generalized and improved result of Chatterjea [25].

4. If $\theta_{2}=\theta_{3}=0$ in Corollary 2.5, we get the generalized and improved result of Berinde [26].

Next, we give the existence theorem of a best proximity point for a generalized $\alpha$-proximal contraction of the second kind.

Theorem 2.6 Let $(X, d)$ be a complete metric space, and let $A$ and $B$ be two non-empty, closed subsets of $X$ such that $A$ is approximately compact with respect to $B$. Assume that $\alpha$ : $A \times A \rightarrow[0, \infty), A_{0}$ and $B_{0}$ are non-empty sets and $T: A \rightarrow B$ is a continuous generalized $\alpha$-proximal contraction of the second kind such that the following conditions hold:

(a) $T$ is $\alpha$-proximal admissible;

(b) $T\left(A_{0}\right) \subseteq B_{0}$;

(c) There exist $x_{0}, x_{1} \in A$ such that $d\left(x_{1}, T x_{0}\right)=d(A, B)$ and $\alpha\left(x_{0}, x_{1}\right) \geq 1$.

Then there exists an element $z \in \mathcal{B}_{\text {est }}(T)$. Further, $T z_{1}=T z_{2}$ whenever $z_{1}, z_{2} \in \mathcal{B}_{\text {est }}(T)$ and $\alpha\left(z_{1}, z_{2}\right) \geq 1$.

Proof Following the arguments in Theorem 2.1, we can construct a sequence $\left\{x_{n}\right\}$ in $A_{0}$ such that

$$
d\left(x_{n}, T x_{n-1}\right)=d(A, B)
$$

and

$$
\alpha\left(x_{n-1}, x_{n}\right) \geq 1
$$

for all $n \in \mathbb{N}$. Since $T$ is a generalized $\alpha$-proximal contraction of the second kind, we have

$$
\begin{aligned}
d\left(T x_{n}, T x_{n+1}\right) \leq & \theta_{1} d\left(T x_{n-1}, T x_{n}\right)+\theta_{2}\left[d\left(T x_{n-1}, T x_{n}\right)+d\left(T x_{n}, T x_{n+1}\right)\right] \\
& +\theta_{3}\left[d\left(T x_{n-1}, T x_{n+1}\right)+d\left(T x_{n}, T x_{n}\right)\right] \\
& +L \min \left\{d\left(T x_{n}, T x_{n}\right), d\left(T x_{n-1}, T x_{n+1}\right),\right. \\
& \left.d\left(T x_{n-1}, T x_{n}\right), d\left(T x_{n}, T x_{n+1}\right)\right\} \\
\leq & \theta_{1} d\left(T x_{n-1}, T x_{n}\right)+\theta_{2}\left[d\left(T x_{n-1}, T x_{n}\right)+d\left(T x_{n}, T x_{n+1}\right)\right] \\
& +\theta_{3}\left[d\left(T x_{n-1}, T x_{n}\right)+d\left(T x_{n}, T x_{n+1}\right)\right]
\end{aligned}
$$

for all $n \in \mathbb{N}$. It follows that $d\left(T x_{n}, T x_{n+1}\right) \leq K d\left(T x_{n-1}, T x_{n}\right)$ for all $n \in \mathbb{N}$, where $K:=$ $\frac{\theta_{1}+\theta_{2}+\theta_{3}}{1-\theta_{2}-\theta_{3}}<1$. Similar to the proof in Theorem 2.1, we obtain that the sequence $\left\{T x_{n}\right\}$ is a 
Cauchy sequence in $B$. Since $B$ is a closed subspace of the complete metric space $X, B$ is complete. Then the sequence $\left\{T x_{n}\right\}$ converges to some $\hat{y} \in B$. Further, we have

$$
\begin{aligned}
d(\hat{y}, A) & \leq d\left(\hat{y}, x_{n+1}\right) \\
& \leq d\left(\hat{y}, T x_{n}\right)+d\left(T x_{n}, x_{n+1}\right) \\
& =d\left(\hat{y}, T x_{n}\right)+d(A, B) \\
& \leq d\left(\hat{y}, T x_{n}\right)+d(\hat{y}, A)
\end{aligned}
$$

for all $n \in \mathbb{N}$. Therefore, $d\left(\hat{y}, x_{n}\right) \rightarrow d(\hat{y}, A)$ as $n \rightarrow \infty$. Since $A$ is approximately compact with respect to $B$, then the sequence $\left\{x_{n}\right\}$ has a subsequence $\left\{x_{n_{k}}\right\}$ converging to some element $z \in A$. Now, using the continuity of $T$, we obtain that $d(z, T z)=$ $\lim _{k \rightarrow \infty} d\left(x_{n_{k}+1}, T x_{n_{k}}\right)=d(A, B)$. Hence $z \in \mathcal{B}_{\text {est }}(T)$.

Finally, we may assume that $z_{1}, z_{2} \in \mathcal{B}_{\text {est }}(T)$ and $\alpha\left(z_{1}, z_{2}\right) \geq 1$. Therefore, we get

$$
\left\{\begin{array}{l}
\alpha\left(z_{1}, z_{2}\right) \geq 1, \\
d\left(z_{1}, T z_{1}\right)=d(A, B), \\
d\left(z_{2}, T z_{2}\right)=d(A, B) .
\end{array}\right.
$$

Since $T$ is a generalized $\alpha$-proximal contraction of the second kind, we have

$$
\begin{aligned}
d\left(T z_{1}, T z_{2}\right) \leq & \theta_{1} d\left(T z_{1}, T z_{2}\right)+\theta_{2}\left[d\left(T z_{1}, T z_{1}\right)+d\left(T z_{2}, T z_{2}\right)\right] \\
& +\theta_{3}\left[d\left(T z_{1}, T z_{2}\right)+d\left(T z_{2}, T z_{1}\right)\right] \\
& +L \min \left\{d\left(T z_{2}, T z_{1}\right), d\left(T z_{1}, T z_{2}\right), d\left(T z_{1}, T z_{1}\right), d\left(T z_{2}, T z_{2}\right)\right\}
\end{aligned}
$$

which implies that

$$
d\left(T z_{1}, T z_{2}\right) \leq \theta_{1}+2 \theta_{3} d\left(T z_{1}, T z_{2}\right)
$$

It follows from $\theta_{1}+2 \theta_{3} \in[0,1)$ that $d\left(T z_{1}, T z_{2}\right)=0$ and hence $T z_{1}=T z_{2}$. This completes the proof.

As a consequence of Theorem 2.6, we state the following corollary.

Corollary 2.7 Let $(X, d)$ be a complete metric space, and let $A$ and $B$ be two non-empty, closed subsets of $X$ such that $A$ is approximately compact with respect to $B$. Assume that $A_{0}$ and $B_{0}$ are non-empty sets and $T: A \rightarrow B$ is continuous such that the following conditions hold:

$\left(\mathrm{a}^{\prime \prime}\right)$ There exist non-negative real numbers $\theta_{1}, \theta_{2}, \theta_{3}$ and $L$ with $\theta_{1}+2 \theta_{2}+2 \theta_{3}<1$ such that for all $u, v, x, y \in A$,

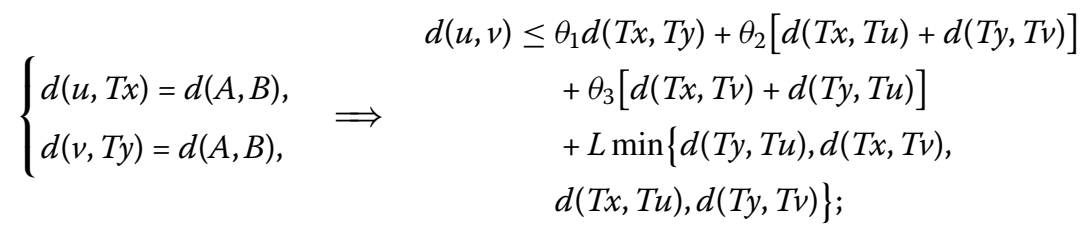


(b) $T\left(A_{0}\right) \subseteq B_{0}$;

(c) There exist $x_{0}, x_{1} \in A$ such that $d\left(x_{1}, T x_{0}\right)=d(A, B)$.

Then there exists an element $z \in \mathcal{B}_{\text {est }}(T)$. Further, $T z_{1}=T z_{2}$ for all $z_{1}, z_{2} \in \mathcal{B}_{\text {est }}(T)$.

Proof By taking $\alpha(x, y)=1$ for all $x, y \in A$ in Theorem 2.6, we get this result.

In Corollary 2.7, if $T$ is a self-mapping, then we get the following fixed point theorem.

Corollary 2.8 Let $(X, d)$ be a complete metric space, $A$ be a non-empty, closed subset of $X$, and let $T: A \rightarrow A$ be a continuous mapping. If there exist non-negative real numbers $\theta_{1}$, $\theta_{2}, \theta_{3}$ and $L$ with $\theta_{1}+2 \theta_{2}+2 \theta_{3}<1$ such that

$$
\begin{aligned}
d(T T x, T T y) \leq & \theta_{1} d(T x, T y)+\theta_{2}[d(T x, T T x)+d(T y, T T y)] \\
& +\theta_{3}[d(T x, T T y)+d(T y, T T x)] \\
& +L \min \{d(T y, T T x), d(T x, T T v), d(T x, T T x), d(T y, T T y)\}
\end{aligned}
$$

for all $x, y \in A$, then $T$ has a unique fixed point.

In the next theorem, we give conditions for the existence of a best proximity point for a non-self mapping that is a generalized $\alpha$-proximal contraction of the first and second kinds. In this theorem, we consider only a completeness hypothesis without assuming the continuity of the non-self mapping and the approximate compactness of the subspace.

Theorem 2.9 Let $(X, d)$ be a complete metric space, and let $A$ and $B$ be two non-empty, closed subsets of $X$. Assume that $\alpha: A \times A \rightarrow[0, \infty), A_{0}$ and $B_{0}$ are non-empty sets and $T: A \rightarrow B$ is a generalized $\alpha$-proximal contraction of the first and second kinds such that the following conditions hold:

(a) $T$ is $\alpha$-proximal admissible;

(b) $T\left(A_{0}\right) \subseteq B_{0}$;

(c) There exist $x_{0}, x_{1} \in A$ such that $d\left(x_{1}, T x_{0}\right)=d(A, B)$ and $\alpha\left(x_{0}, x_{1}\right) \geq 1$;

(d) If $\left\{x_{n}\right\}$ is a sequence in $A$ such that $\alpha\left(x_{n}, x_{n+1}\right) \geq 1$ for all $n \in \mathbb{N}$ and $x_{n} \rightarrow x$ as $n \rightarrow \infty$ for some $x \in A$, then $\alpha\left(x_{n}, x\right) \geq 1$ for all $n \in \mathbb{N}$.

Then there exists an element $z \in A_{0}$ such that $d(z, T z)=d(A, B)$, that is, $T$ has at least one best proximity point. Moreover, if $\alpha\left(z_{1}, z_{2}\right) \geq 1$ for all $z_{1}, z_{2} \in \mathcal{B}_{\text {est }}(T)$, then $T$ has a unique best proximity point.

Proof Following the arguments in Theorem 2.1, we can construct a sequence $\left\{x_{n}\right\}$ in $A_{0}$ such that

$$
d\left(x_{n}, T x_{n-1}\right)=d(A, B)
$$

and

$$
\alpha\left(x_{n-1}, x_{n}\right) \geq 1
$$

for all $n \in \mathbb{N}$. 
Also, using similar arguments as in the proof of Theorem 2.1, we conclude that the sequence $\left\{x_{n}\right\}$ is a Cauchy sequence, and hence converges to some $z \in A$. Moreover, on the lines of Theorem 2.6, we obtain that the sequence $\left\{T x_{n}\right\}$ is a Cauchy sequence and hence converges to some $\hat{y} \in B$. Therefore, we have $d(z, \hat{y})=\lim _{n \rightarrow \infty} d\left(x_{n+1}, T x_{n}\right)=$ $d(A, B)$, hence $z$ must be in $A_{0}$. Since $T\left(A_{0}\right) \subseteq B_{0}$, then $d(\hat{u}, T z)=d(A, B)$ for some $\hat{u} \in A$.

From the assumption (d) and (2.12), we have

$$
\alpha\left(x_{n}, z\right) \geq 1
$$

for all $n \in \mathbb{N}$.

Now we have

$$
\left\{\begin{array}{l}
\alpha\left(x_{n}, z\right) \geq 1 \\
d\left(x_{n+1}, T x_{n}\right)=d(A, B), \\
d(\hat{u}, T z)=d(A, B)
\end{array}\right.
$$

for all $n \in \mathbb{N}$. Using the fact that $T$ is a generalized $\alpha$-proximal contraction of the first kind, we have

$$
\begin{aligned}
d\left(x_{n+1}, \hat{u}\right) \leq & \theta_{1} d\left(x_{n}, z\right)+\theta_{2}\left[d\left(x_{n}, x_{n+1}\right)+d(z, \hat{u})\right]+\theta_{3}\left[d\left(x_{n}, \hat{u}\right)+d\left(z, x_{n+1}\right)\right] \\
& +L \min \left\{d\left(z, x_{n+1}\right), d\left(x_{n}, \hat{u}\right), d\left(x_{n}, x_{n+1}\right), d(z, \hat{u})\right\}
\end{aligned}
$$

for all $n \in \mathbb{N}$. Taking the limit as $n \rightarrow \infty$ in the previous inequality, we have

$$
d(z, \hat{u}) \leq\left(\theta_{2}+\theta_{3}\right) d(z, \hat{u}) .
$$

Since $\theta_{2}+\theta_{3} \in[0,1)$, we get $d(z, \hat{u})=0$ and then $z=\hat{u}$. Thus, it follows that $d(z, T z)=$ $d(\hat{u}, T z)=d(A, B)$. Therefore, $z \in \mathcal{B}_{\text {est }}(T)$.

For the uniqueness of a best proximity point of $T$, we proceed similarly to the proof of Theorem 2.1. Then, in order to avoid repetition, the details are omitted.

Corollary 2.10 Let $(X, d)$ be a complete metric space, and let $A$ and $B$ be two non-empty, closed subsets of $X$. Assume that $A_{0}$ and $B_{0}$ are non-empty sets and $T: A \rightarrow B$ satisfies the following conditions:

$\left(\mathrm{a}^{\prime}\right)$ There exist non-negative real numbers $\theta_{1}, \theta_{2}, \theta_{3}$ and $L$ with $\theta_{1}+2 \theta_{2}+2 \theta_{3}<1$ such that for all $u, v, x, y \in A$,

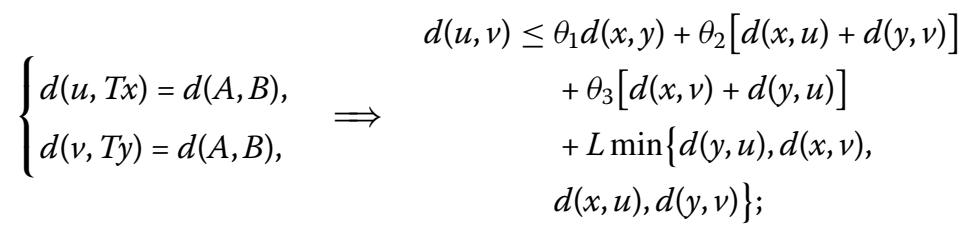


$\left(\mathrm{a}^{\prime \prime}\right)$ There exist non-negative real numbers $\bar{\theta}_{1}, \bar{\theta}_{2}, \bar{\theta}_{3}$ and $\bar{L}$ with $\bar{\theta}_{1}+2 \bar{\theta}_{2}+2 \bar{\theta}_{3}<1$ such that for all $u, v, x, y \in A$,

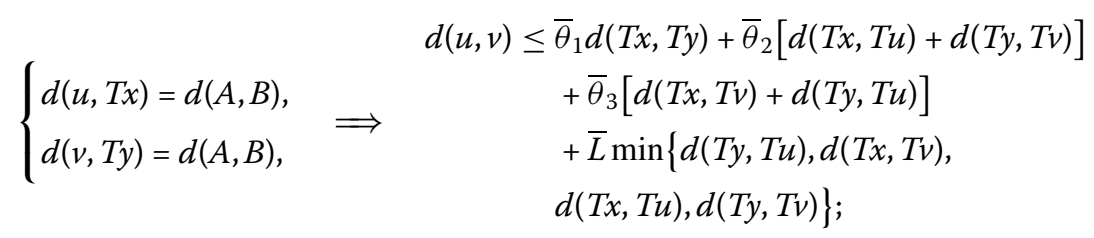

(b) $T\left(A_{0}\right) \subseteq B_{0}$;

(c) There exist $x_{0}, x_{1} \in A$ such that $d\left(x_{1}, T x_{0}\right)=d(A, B)$.

Then there exists a unique $z \in A_{0}$ such that $d(z, T z)=d(A, B)$, that is, $T$ has a unique best proximity point.

Proof By taking $\alpha(x, y)=1$ for all $x, y \in A$ in Theorem 2.9, we get this result.

\section{Best proximity point theorems on a metric space endowed with an arbitrary binary relation}

In this section, we apply our results in the previous section (Theorems 2.1, 2.6 and 2.9) to the best proximity point theorems on a metric space endowed with an arbitrary binary relation. Moreover, we obtain a special case of these results as corollaries in partially ordered metric spaces.

Before presenting our results, we need a few preliminaries. Let $(X, d)$ be a metric space and $\mathcal{R}$ be an arbitrary binary relation over $X$. Denote

$$
\mathcal{S}:=\mathcal{R} \cup \mathcal{R}^{-1}
$$

this is the symmetric relation attached to $R$. Clearly,

$$
x, y \in X, \quad x \mathcal{S} y \quad \Longleftrightarrow \quad x \mathcal{R} y \quad \text { or } y \mathcal{R} x .
$$

Definition 3.1 Let $(X, d)$ be a metric space, let $\mathcal{R}$ be a binary relation over $X$, and let $A$ and $B$ be two non-empty subsets of $X$. We say that $T: A \rightarrow B$ is a proximal comparative mapping if

$$
\left.\begin{array}{l}
x \mathcal{S} y, \\
d(u, T x)=d(A, B), \\
d(v, T y)=d(A, B),
\end{array}\right\} \Rightarrow u \mathcal{S} v
$$

for all $u, v, x, y \in A$.

We have the following best proximity point result.

Theorem 3.1 Let $(X, d)$ be a complete metric space, let $\mathcal{R}$ be a binary relation over $X$, and let $A$ and $B$ be two non-empty, closed subsets of $X$ such that $B$ is approximately compact with respect to $A$. Assume that $A_{0}$ and $B_{0}$ are non-empty sets and $T: A \rightarrow B$ such that the following conditions hold: 
(A) $T$ is proximal comparative mapping;

(A') There exist non-negative real numbers $\theta_{1}, \theta_{2}, \theta_{3}$ and $L$ with $\theta_{1}+2 \theta_{2}+2 \theta_{3}<1$ such that for all $u, v, x, y \in A$,

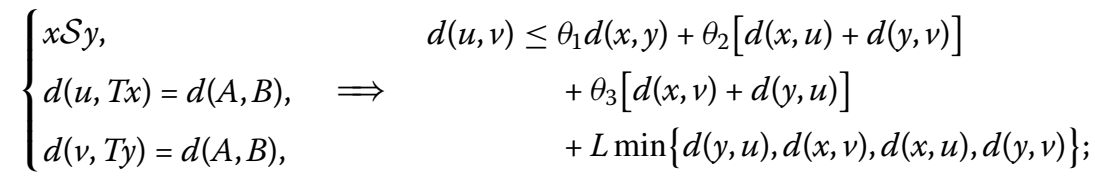

(B) $T\left(A_{0}\right) \subseteq B_{0}$;

(C) There exist $x_{0}, x_{1} \in A$ such that $d\left(x_{1}, T x_{0}\right)=d(A, B)$ and $x_{0} \mathcal{S} x_{1}$;

(D) If $\left\{x_{n}\right\}$ is a sequence in $A$ such that $x_{n} \mathcal{S} x_{n+1}$ for all $n \in \mathbb{N}$ and $x_{n} \rightarrow x$ as $n \rightarrow \infty$ for some $x \in A$, then $x_{n} \mathcal{S} x$ for all $n \in \mathbb{N}$.

Then there exists an element $z \in A_{0}$ such that $d(z, T z)=d(A, B)$, that is, $T$ has at least one best proximity point. Moreover, if $z_{1} \mathcal{S} z_{2}$ for all $z_{1}, z_{2} \in \mathcal{B}_{\text {est }}(T)$, then $T$ has a unique best proximity point.

Proof Define the mapping $\alpha: A \times A \rightarrow[0, \infty)$ by

$$
\alpha(x, y)= \begin{cases}1, & x \mathcal{S} y \\ 0, & \text { otherwise }\end{cases}
$$

Since $T$ is a proximal comparative mapping, we have

$$
\begin{aligned}
& \left.\begin{array}{l}
x \mathcal{S} y, \\
d(u, T x)=d(A, B),
\end{array}\right\} \Rightarrow u \mathcal{S} v \\
& d(v, T y)=d(A, B),
\end{aligned}
$$

for all $u, v, x, y \in A$. By the definition of $\alpha$, we obtain that

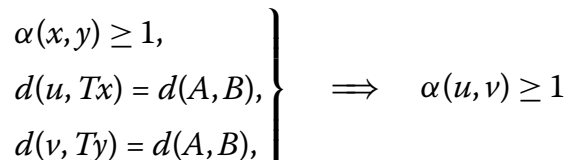

for all $u, v, x, y \in A$. This shows that $T$ is $\alpha$-proximal admissible.

From $\left(A^{\prime}\right)$ and the construction of mapping $\alpha$, we get

$$
\left\{\begin{array}{lrl}
\alpha(x, y) \geq 1, & d(u, v) \leq & \theta_{1} d(x, y)+\theta_{2}[d(x, u)+d(y, v)] \\
d(u, T x)=d(A, B), & & +\theta_{3}[d(x, v)+d(y, u)] \\
d(v, T y)=d(A, B), & &
\end{array}\right.
$$

for all $u, v, x, y \in A$, that is, $T$ is a generalized $\alpha$-proximal contraction of the first kind.

Since condition (C) implies that $d\left(x_{1}, T x_{0}\right)=d(A, B)$ and $\alpha\left(x_{0}, x_{1}\right) \geq 1$, condition (c) in Theorem 2.1 holds. It is easy to see that condition (D) implies condition (d) in Theorem 2.1.

Now, all the hypotheses of Theorem 2.1 are satisfied, and the desired result follows immediately from this theorem. 
Theorem 3.2 Let $(X, d)$ be a complete metric space, let $\mathcal{R}$ be a binary relation over $X$, and let $A$ and $B$ be two non-empty, closed subsets of $X$ such that $A$ is approximately compact with respect to $B$. Assume that $A_{0}$ and $B_{0}$ are non-empty and $T: A \rightarrow B$ is continuous such that the following conditions hold:

(A) $T$ is a proximal comparative mapping;

(A") There exist non-negative real numbers $\theta_{1}, \theta_{2}, \theta_{3}$ and $L$ with $\theta_{1}+2 \theta_{2}+2 \theta_{3}<1$ such that for all $u, v, x, y \in A$,

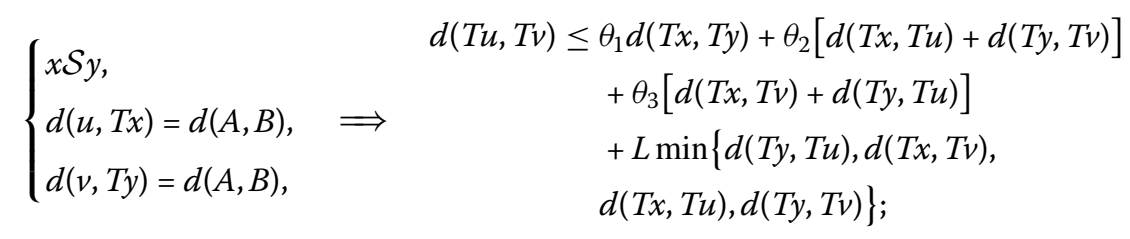

(b) $T\left(A_{0}\right) \subseteq B_{0}$;

(c) There exist $x_{0}, x_{1} \in A$ such that $d\left(x_{1}, T x_{0}\right)=d(A, B)$ and $x_{0} \mathcal{S} x_{1}$.

Then there exists an element $z \in \mathcal{B}_{\text {est }}(T)$. Further, $T z_{1}=T z_{2}$ whenever $z_{1}, z_{2} \in \mathcal{B}_{\text {est }}(T)$ and $z_{1} \mathcal{S} z_{2}$.

Proof The result follows from Theorem 2.6 by considering the mapping $\alpha$ as in the proof of Theorem 3.1.

Theorem 3.3 Let $(X, d)$ be a complete metric space, let $\mathcal{R}$ be a binary relation over $X$, and let $A$ and $B$ be two non-empty, closed subsets of $X$. Assume that $A_{0}$ and $B_{0}$ are non-empty sets and $T: A \rightarrow B$ such that the following conditions hold:

(A) $T$ is a proximal comparative mapping;

(A') There exist non-negative real numbers $\theta_{1}, \theta_{2}, \theta_{3}$ and $L$ with $\theta_{1}+2 \theta_{2}+2 \theta_{3}<1$ such that for all $u, v, x, y \in A$,

$$
\left\{\begin{array}{llrl}
x \mathcal{S} y, & d(u, v) \leq & \theta_{1} d(x, y)+\theta_{2}[d(x, u)+d(y, v)] \\
d(u, T x)=d(A, B), & & +\theta_{3}[d(x, v)+d(y, u)] \\
d(v, T y)=d(A, B), & & & L \min \{d(y, u), d(x, v), d(x, u), d(y, v)\}
\end{array}\right.
$$

$\left(\mathrm{A}^{\prime \prime}\right)$ There exist non-negative real numbers $\bar{\theta}_{1}, \bar{\theta}_{2}, \bar{\theta}_{3}$ and $L$ with $\bar{\theta}_{1}+2 \bar{\theta}_{2}+2 \bar{\theta}_{3}<1$ such that for all $u, v, x, y \in A$,

$$
\left\{\begin{array}{lcl}
x \mathcal{S} y, & d(T u, T v) \leq & \bar{\theta}_{1} d(T x, T y)+\bar{\theta}_{2}[d(T x, T u)+d(T y, T v)] \\
d(u, T x)=d(A, B), & +\bar{\theta}_{3}[d(T x, T v)+d(T y, T u)] \\
d(v, T y)=d(A, B), & +L \min \{d(T y, T u), d(T x, T v), \\
& d(T x, T u), d(T y, T v)\} ;
\end{array}\right.
$$

(B) $T\left(A_{0}\right) \subseteq B_{0}$;

(C) There exist $x_{0}, x_{1} \in A$ such that $d\left(x_{1}, T x_{0}\right)=d(A, B)$ and $x_{0} \mathcal{S} x_{1}$;

(D) If $\left\{x_{n}\right\}$ is a sequence in $A$ such that $x_{n} \mathcal{S} x_{n+1}$ for all $n \in \mathbb{N}$ and $x_{n} \rightarrow x$ as $n \rightarrow \infty$ for some $x \in A$, then $x_{n} \mathcal{S} x$ for all $n \in \mathbb{N}$. 
Then there exists an element $z \in A_{0}$ such that $d(z, T z)=d(A, B)$, that is, $T$ has at least one best proximity point. Moreover, if $z_{1} \mathcal{S} z_{2}$ for all $z_{1}, z_{2} \in \mathcal{B}_{\text {est }}(T)$, then $T$ has a unique best proximity point.

Proof This result follows from Theorem 2.9 by considering the mapping $\alpha$ given in the proof of Theorem 3.1.

Next, we deduce Theorems 3.1, 3.2 and 3.3 to the special case in the context of partially ordered metric spaces. Before studying the next results, we give the following definitions.

Definition 3.2 Let $X$ be a non-empty set. Then $(X, d, \preceq)$ is called a partially ordered metric space if $(X, d)$ is a metric space and $(X, \preceq)$ is a partially ordered set.

For the partially ordered set $(X, \preceq)$, we define

$$
\asymp:=\{(x, y) \in X \times X: x \preceq y \text { or } y \preceq x\} .
$$

Definition 3.3 Let $(X, d, \preceq)$ be a partially ordered metric space, and let $A$ and $B$ be two non-empty subsets of $X$. We say that $T: A \rightarrow B$ is a proximal comparative mapping with respect to $\preceq$ if

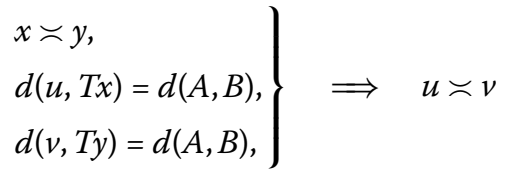

for all $u, v, x, y \in A$.

It is obtained that $\preceq$ is a binary operation on $X$ and $\asymp=\preceq U \preceq^{-1}$. Therefore, we get the following best proximity point results in a partially ordered metric space.

Corollary 3.4 Let $(X, d, \preceq)$ be a complete partially ordered metric space, and let $A$ and $B$ be two non-empty, closed subsets of $X$ such that $B$ is approximately compact with respect to $A$. Assume that $A_{0}$ and $B_{0}$ are non-empty sets and $T: A \rightarrow B$ such that the following conditions hold:

(A) $T$ is proximal comparative with respect to $\preceq$;

(A') There exist non-negative real numbers $\theta_{1}, \theta_{2}, \theta_{3}$ and $L$ with $\theta_{1}+2 \theta_{2}+2 \theta_{3}<1$ such that for all $u, v, x, y \in A$,

$$
\left\{\begin{array}{llrl}
x \asymp y, & & d(u, v) \leq & \theta_{1} d(x, y)+\theta_{2}[d(x, u)+d(y, v)] \\
d(u, T x)=d(A, B), & & +\theta_{3}[d(x, v)+d(y, u)] \\
d(v, T y)=d(A, B), & & & L \min \{d(y, u), d(x, v), d(x, u), d(y, v)\}
\end{array}\right.
$$

(B) $T\left(A_{0}\right) \subseteq B_{0}$;

(C) There exist $x_{0}, x_{1} \in A$ such that $d\left(x_{1}, T x_{0}\right)=d(A, B)$ and $x_{0} \asymp x_{1}$;

(D) If $\left\{x_{n}\right\}$ is a sequence in $A$ such that $x_{n} \asymp x_{n+1}$ for all $n \in \mathbb{N}$ and $x_{n} \rightarrow x$ as $n \rightarrow \infty$ for some $x \in A$, then $x_{n} \asymp x$ for all $n \in \mathbb{N}$. 
Then there exists an element $z \in A_{0}$ such that $d(z, T z)=d(A, B)$, that is, $T$ has at least one best proximity point. Moreover, if $z_{1} \asymp z_{2}$ for all $z_{1}, z_{2} \in \mathcal{B}_{\text {est }}(T)$, then $T$ has a unique best proximity point.

Corollary 3.5 Let $(X, d, \preceq)$ be a complete partially ordered metric space, and let $A$ and $B$ be two non-empty, closed subsets of $X$ such that $A$ is approximately compact with respect to $B$. Assume that $A_{0}$ and $B_{0}$ are non-empty sets and $T: A \rightarrow B$ is continuous such that the following conditions hold:

(A) $T$ is a proximal comparative mapping with respect to $\preceq$;

$\left(\mathrm{A}^{\prime \prime}\right)$ There exist non-negative real numbers $\theta_{1}, \theta_{2}, \theta_{3}$ and $L$ with $\theta_{1}+2 \theta_{2}+2 \theta_{3}<1$ such that for all $u, v, x, y \in A$,

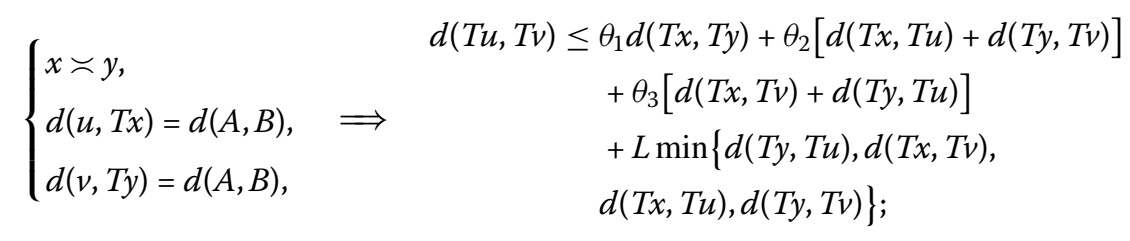

(B) $T\left(A_{0}\right) \subseteq B_{0}$;

(C) There exist $x_{0}, x_{1} \in A$ such that $d\left(x_{1}, T x_{0}\right)=d(A, B)$ and $x_{0} \asymp x_{1}$.

Then there exists an element $z \in \mathcal{B}_{\text {est }}(T)$. Further, $T z_{1}=T z_{2}$ whenever $z_{1}, z_{2} \in \mathcal{B}_{\text {est }}(T)$ and $z_{1} \asymp z_{2}$.

Corollary 3.6 Let $(X, d, \preceq)$ be a complete partially ordered metric space, and let $A$ and $B$ be two non-empty, closed subsets of $X$. Assume that $A_{0}$ and $B_{0}$ are non-empty sets and $T: A \rightarrow B$ such that the following conditions hold:

(A) $T$ is a proximal comparative mapping with respect to $\preceq$;

(A') There exist non-negative real numbers $\theta_{1}, \theta_{2}, \theta_{3}$ and $L$ with $\theta_{1}+2 \theta_{2}+2 \theta_{3}<1$ such that for all $u, v, x, y \in A$,

$$
\left\{\begin{array}{llc}
x \asymp y, & d(u, v) \leq & \theta_{1} d(x, y)+\theta_{2}[d(x, u)+d(y, v)] \\
d(u, T x)=d(A, B), & & +\theta_{3}[d(x, v)+d(y, u)] \\
d(v, T y)=d(A, B), & &
\end{array}\right.
$$

$\left(\mathrm{A}^{\prime \prime}\right)$ There exist non-negative real numbers $\bar{\theta}_{1}, \bar{\theta}_{2}, \bar{\theta}_{3}$ and $L$ with $\bar{\theta}_{1}+2 \bar{\theta}_{2}+2 \bar{\theta}_{3}<1$ such that for all $u, v, x, y \in A$,

$$
\begin{cases}x \asymp y, & d(T u, T v) \leq \\ & \bar{\theta}_{1} d(T x, T y)+\bar{\theta}_{2}[d(T x, T u)+d(T y, T v)] \\ d(u, T x)=d(A, B), & +\bar{\theta}_{3}[d(T x, T v)+d(T y, T u)] \\ d(v, T y)=d(A, B), & +L \min \{d(T y, T u), d(T x, T v), \\ & d(T x, T u), d(T y, T v)\} ;\end{cases}
$$

(B) $T\left(A_{0}\right) \subseteq B_{0}$;

(C) There exist $x_{0}, x_{1} \in A$ such that $d\left(x_{1}, T x_{0}\right)=d(A, B)$ and $x_{0} \asymp x_{1}$; 
(D) If $\left\{x_{n}\right\}$ is a sequence in $A$ such that $x_{n} \asymp x_{n+1}$ for all $n \in \mathbb{N}$ and $x_{n} \rightarrow x$ as $n \rightarrow \infty$ for some $x \in A$, then $x_{n} \asymp x$ for all $n \in \mathbb{N}$.

Then there exists an element $z \in A_{0}$ such that $d(z, T z)=d(A, B)$, that is, $T$ has at least one best proximity point. Moreover, if $z_{1} \asymp z_{2}$ for all $z_{1}, z_{2} \in \mathcal{B}_{\text {est }}(T)$, then $T$ has a unique best proximity point.

\section{Remarks and conclusions}

Recently, Salimi et al. [21] presented some fixed point results by modifying $\alpha-\psi$ contraction types in [16] and [29].

Definition 4.1 [21] Let $G: X \rightarrow X$ be a mapping on a metric space $(X, d)$, and let $\alpha, \eta$ : $X \times X \rightarrow[0, \infty)$ be two functions. We say that $G$ is an $\alpha$-admissible mapping with respect to $\eta$ if for $x, y \in X$, we have

$$
\alpha(x, y) \geq \eta(x, y) \quad \Rightarrow \quad \alpha(G x, G y) \geq \eta(G x, G y) .
$$

Inspired by Salimi et al. [21] one can suggest the following definitions.

Definition 4.2 Let $A$ and $B$ be two non-empty subsets of a metric space $(X, d)$ and $\alpha, \beta$ : $A \times A \rightarrow[0, \infty)$. A mapping $T: A \rightarrow B$ is said to be an $(\alpha, \beta)$-proximal contraction of the first kind if there exist non-negative real numbers $\theta_{1}, \theta_{2}, \theta_{3}$ and $L$ with $\theta_{1}+2 \theta_{2}+2 \theta_{3}<1$ such that for all $u, v, x, y \in A$,

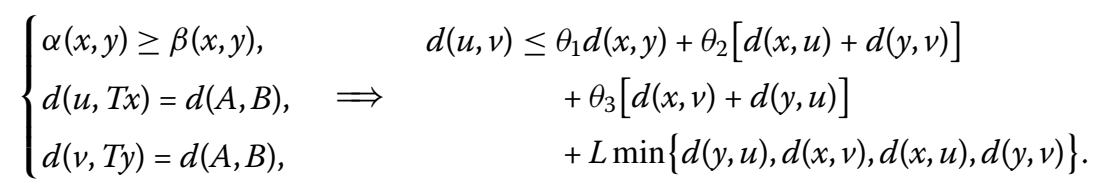

Definition 4.3 Let $A$ and $B$ be two non-empty subsets of a metric space $(X, d)$ and $\alpha, \beta$ : $A \times A \rightarrow[0, \infty)$. A mapping $T: A \rightarrow B$ is said to be an $(\alpha, \beta)$-proximal contraction of the second kind if there exist non-negative real numbers $\theta_{1}, \theta_{2}, \theta_{3}$ and $L$ with $\theta_{1}+2 \theta_{2}+2 \theta_{3}<1$ such that for all $u, v, x, y \in A$,

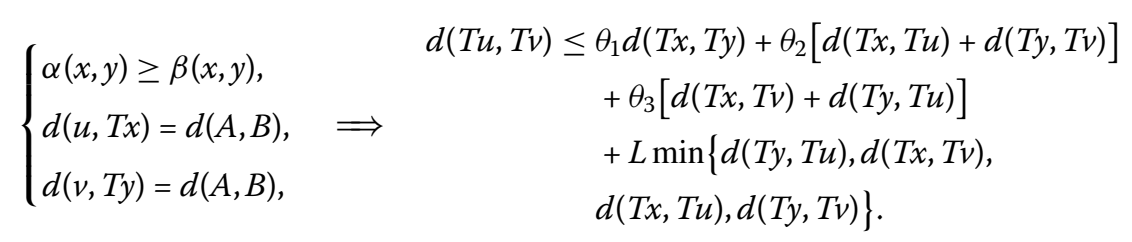

By replacing an $(\alpha, \beta)$-proximal contraction of the first kind with an $\alpha$-proximal contraction of the first kind (that is, by replacing Definition 4.2 with Definition 2.1) in the statements of Theorem 2.1, one can get the following theorem.

Theorem 4.1 Let $(X, d)$ be a complete metric space, and let $A$ and $B$ be two non-empty, closed subsets of $X$ such that $B$ is approximately compact with respect to $A$. Assume that $\alpha, \beta: A \times A \rightarrow[0, \infty), A_{0}$ and $B_{0}$ are non-empty sets and $T: A \rightarrow B$ is an $(\alpha, \beta)$-proximal contraction of the first kind such that the following conditions hold: 
(a) $T$ is $\alpha$-proximal admissible with respect to $\beta$;

(b) $T\left(A_{0}\right) \subseteq B_{0}$;

(c) There exist $x_{0}, x_{1} \in A$ such that $d\left(x_{1}, T x_{0}\right)=d(A, B)$ and $\alpha\left(x_{0}, x_{1}\right) \geq \beta\left(x_{0}, x_{1}\right)$;

(d) If $\left\{x_{n}\right\}$ is a sequence in $A$ such that $\alpha\left(x_{n}, x_{n+1}\right) \geq \beta\left(x_{n}, x_{n+1}\right)$ for all $n \in \mathbb{N}$ and $x_{n} \rightarrow x$ as $n \rightarrow \infty$ for some $x \in A$, then $\alpha\left(x_{n}, x\right) \geq \beta\left(x_{n}, x\right)$ for all $n \in \mathbb{N}$.

Then there exists an element $z \in A_{0}$ such that $d(z, T z)=d(A, B)$, that is, $T$ has at least one best proximity point. Moreover, if $\alpha\left(z_{1}, z_{2}\right) \geq \beta\left(z_{1}, z_{2}\right)$ for all $z_{1}, z_{2} \in \mathcal{B}_{\text {est }}(T)$, then $T$ has a unique best proximity point.

In this case, the proof is the analog of the proof of Theorem 2.1. In the same setting, the analogues of Theorem 2.6, Theorem 2.9, Theorem 3.1, Theorem 3.2, Theorem 3.3 can be obtained easily.

Very recently, Berzig and Karapınar [30] proved that the first main result of Salimi et al. [21] follows from a result of Karapinar and Samet [29]. Thus, Definition 4.2 and Definition 4.3 can be considered as a consequence of Definition 2.1 and Definition 2.2.

\section{Competing interests}

The authors declare that they have no competing interests.

\section{Authors' contributions}

All authors contributed equally and significantly in writing this paper. All authors read and approved the final manuscript.

\section{Acknowledgements}

The authors thank reviewers for their remarkable comments, suggestion and ideas that helped to improve this paper

Received: 24 June 2013 Accepted: 4 November 2013 Published: 27 Nov 2013

\section{References}

1. Banach, S: Sur les opérations dans les ensembles abstraits et leurs applications aux équations intégrales. Fundam. Math. 3, 133-181 (1922)

2. Fan, K: Extensions of two fixed point theorems of F. E. Browder. Math. Z. 112, 234-240 (1969)

3. Jleli, M, Karapınar, E, Samet, B: On best proximity points under the P-property on partially ordered metric spaces. Abstr. Appl. Anal. 2013, Article ID 150970 (2013)

4. Jleli, M, Karapınar, E, Samet, B: Best proximity points for generalized $\alpha$ - $\psi$-proximal contractive type mappings. J. Appl. Math. 2013, Article ID 534127 (2013)

5. Jleli, M, Karapınar, E, Samet, B: Best proximity point results for MK-proximal contractions. Abstr. Appl. Anal. 2012 Article ID 193085 (2012)

6. Karapınar, E: Best proximity points of Kannan type cyclic weak $\phi$-contractions in ordered metric spaces. An. Univ. "Ovidius" Constanţa, Ser. Mat. 20(3), 51-64 (2012)

7. Karapınar, E: Best proximity points of cyclic mappings. Appl. Math. Lett. 25(11), 1761-1766 (2012)

8. Karapınar, E: On best proximity point of $\psi$-Geraghty contractions. Fixed Point Theory Appl. 2013, 200 (2013)

9. Kumam, P, Aydi, H, Karapınar, E, Sintunavarat, W: Best proximity points and extension of Mizoguchi-Takahashi's fixed point theorems. Fixed Point Theory Appl. 2013, 242 (2013)

10. Mongkolkeha, C, Cho, YJ, Kumam, P: Best proximity points for generalized proximal C-contraction mappings in metric spaces with partial orders. J. Inequal. Appl. 2013, 94 (2013)

11. Mongkolkeha, C, Cho, YJ, Kumam, P: Best proximity points for Geraghty's proximal contraction mapping mappings. Fixed Point Theory Appl. 2013, 180 (2013)

12. Sanhan, W, Mongkolkeha, C, Kumam, P: Generalized proximal $\psi$-contraction mappings and best proximity points. Abstr. Appl. Anal. 2012, Article ID 896912 (2012)

13. Sintunavarat, W, Kumam, P: Coupled best proximity point theorem in metric spaces. Fixed Point Theory Appl. 2012, 93 (2012)

14. Sintunavarat, W, Kumam, P: The existence theorems of an optimal approximate solution for generalized proximal contraction mappings. Abstr. Appl. Anal. 2013, Article ID 375604 (2013)

15. Efimov, NV, Stechkin, SB: Approximative compactness and Chebyshev sets. Dokl. Akad. Nauk SSSR 140, 522-524 (1961) (in Russian)

16. Samet, B, Vetro, C, Vetro, P: Fixed point theorems for $\alpha$ - $\psi$-contractive type mappings. Nonlinear Anal. 75, 2154-2165 (2012)

17. Agarwal, RP, Sintunavarat, W, Kumam, P: PPF dependent fixed point theorems for an $\alpha_{c}$-admissible non-self mapping in the Razumikhin class. Fixed Point Theory Appl. 2013, 280 (2013)

18. Alghamdi, MA, Karapınar, E: G- $\beta-\psi$ contractive type mappings and related fixed point theorems. J. Inequal. Appl. 2013, 70 (2013)

19. Hussain, N, Karapınar, E, Salimi, P, Vetro, P: Fixed point results for $G^{m}$-Meir-Keeler contractive and G- $(\alpha, \psi)$-Meir-Keeler contractive mappings. Fixed Point Theory Appl. 2013, 34 (2013) 
20. Kutbi, MA, Sintunavarat, W: The existence of fixed point theorems via $w$-distance and $\alpha$-admissible mappings and applications. Abstr. Appl. Anal. 2013, Article ID 165434 (2013)

21. Salimi, P, Latif, A, Hussain, N: Modified $\alpha$ - $\psi$-contractive mappings with applications. Fixed Point Theory Appl. 2013 $151(2013)$

22. Sintunavarat, W, Plubtieng, S, Katchang, P: Fixed point result and applications on b-metric space endowed with an arbitrary binary relation. Fixed Point Theory Appl. 2013, 296 (2013). doi:10.1186/1687-1812-2013-296

23. Jleli, M, Samet, B: Best proximity points for $\alpha$ - $\psi$-proximal contractive type mappings and applications. Bull. Sci. Math. (2013). doi:10.1016/j.bulsci.2013.02.003

24. Kannan, R: Some results on fixed points II. Am. Math. Mon. 76, 405-408 (1969)

25. Chatterjea, SK: Fixed point theorems. C. R. Acad. Bulgare Sci. 25, 727-730 (1972)

26. Berinde, V: Approximating fixed points of weak contractions using the Picard iteration. Nonlinear Anal. Forum 9(1), 43-53 (2004)

27. Kirk, WA, Reich, S, Veeramani, P: Proximinal retracts and best proximity pair theorems. Numer. Funct. Anal. Optim. 24 851-862 (2003)

28. Basha, SS, Veeramani, P: Best proximity pair theorems for multifunctions with open fibres. J. Approx. Theory 103 , 119-129 (2000)

29. Karapınar, E, Samet, B: Generalized $\alpha$ - $\psi$-contractive type mappings and related fixed point theorems with applications. Abstr. Appl. Anal. 2012, Article ID 793486 (2012)

30. Berzig, M, Karapınar, E: Note on "Modified $\alpha$ - $\psi$-contractive mappings with application". Preprint

10.1186/1687-1812-2013-323

Cite this article as: Karapınar and Sintunavarat: The existence of optimal approximate solution theorems for generalized $\alpha$-proximal contraction non-self mappings and applications. Fixed Point Theory and Applications 2013, 2013:323

\section{Submit your manuscript to a SpringerOpen ${ }^{\circ}$ journal and benefit from:}

- Convenient online submission

- Rigorous peer review

- Immediate publication on acceptance

- Open access: articles freely available online

- High visibility within the field

- Retaining the copyright to your article 